\title{
Immunogenicity of an Inactivated Canine Adenovirus Type 1 Vaccine for Foxes
}

\section{OPEN ACCESS}

Edited by:

Levon Abrahamyan,

Université de Montréal, Canada

Reviewed by:

Madhuri Subbiah,

National Institute of Animal

Biotechnology (NIAB), India

Francisco Rivera-Benítez,

Instituto Nacional de Investigaciones

Forestales, Agrícolas y Pecuarias

(INIFAP), Mexico

*Correspondence:

Yanzhu Zhu

zyzzu@126.com

tThese authors have contributed equally to this work

Specialty section:

This article was submitted to Veterinary Infectious Diseases,

a section of the journal

Frontiers in Veterinary Science

Received: 11 March 2021

Accepted: 13 January 2022

Published: 15 February 2022

Citation:

Fu Y, Sun J, Lian S, Deng X, Zhang L, Shao J, Yu H, Yan X and Zhu Y (2022) Immunogenicity of an Inactivated

Canine Adenovirus Type 1 Vaccine for Foxes. Front. Vet. Sci. 9:678671. doi: 10.3389/fvets.2022.678671

\author{
Yang Fu ${ }^{1,2+}$, Jie Sun ${ }^{1+}$, Shizhen Lian ${ }^{1}$, Xiaoyu Deng ${ }^{1}$, Lei Zhang ${ }^{1}$, Jikai Shao ${ }^{3}$, \\ Hongguang $\mathrm{Yu}^{4}$, Xijun Yan ${ }^{1}$ and Yanzhu Zhu ${ }^{1 *}$
}

${ }^{1}$ Institute of Special Animal and Plant Sciences, Chinese Academy of Agricultural Sciences, Changchun, China, ${ }^{2}$ Department of Food Science and Chemical Engineering, Veterinary Medicine, Heze Vocational College Heze, Heze, China, ${ }^{3}$ Yuncheng No. 1 Middle School of Shandong Province, Heze, China, ${ }^{4}$ The Second Experimental Ocean School of Jilin Province, Jilin, China

Canine adenovirus type 1 (CAdV-1) is the etiologic agent of fox encephalitis. As with most viral agents, the best method of prevention is vaccination. In this study, the CAdV-1 strain F1301 strain was used to construct a new type 1 canine adenovirus inactivated vaccine candidate, and its safety and immunogenicity were evaluated in silver foxes. Next, animals were challenged and survival rates of animals vaccinated with either the commercially available or the current candidate vaccine were examined. The results confirmed that the inactivated CAdV-1 vaccine prepared in this study can effectively protect against challenge with virulent CAdV-1 in silver foxes, and the safety profile was improved relative to that of the commercial vaccine. This study confirmed that the fox CAdV-1 F1301 strain can be used as a platform for an inactivated CAdV-1 vaccine.

Keywords: canine adenovirus type 1, silver fox, inactivated vaccine, immunogenicity, CAdV-1 F1301 strain

\section{INTRODUCTION}

Canine adenoviruses belong to the mastadenovirus genus of mammalian adenoviruses and the Adenoviridae family, which are divided into canine adenovirus type 1 (CAdV-1) and canine adenovirus type 2 (CAdV-2). Genomic sequence homology between CAdV-1 and CAdV-2 is about $70 \%$ (1). Although structurally similar, CAdV-1 and CAdV-2 infect different tissues. CAdV-1 targets the digestive tract and causes infectious hepatitis, nephritis, encephalitis, and conjunctivitis (2). Conversely, CAdV-2 infects respiratory tissues and induces infectious laryngotracheitis (3). CAdV-1 infects a wide range of animals, including dogs, foxes, wolves (4), mountain dogs, bears, skunks, and guinea pigs. The virus consists of a non-enveloped icosahedral virion of $80 \mathrm{~nm}$ in diameter that replicates in the nucleus of the host cell. The CAdV-1 genome is double-stranded DNA approximately $31 \mathrm{~kb}$ in size. The morphological and structural characteristics of CAdV-1 are typical of adenoviruses. The protein capsid is composed of 252 subunits, including the hexons, pentons, and fibrils. The hexons are the major component of the viral capsid, which also provide the complement activating epitopes. from the pentons extend a filament capped with a ball structure, the surface of which is coated with hemagglutinin molecules. When the virus infects the host cell, the ball functions as the receptor binding protein to initiate fusion and entry.

CAdV-1 is the etiologic agent of fox encephalitis. Typical symptoms of CAdV-1 infection in acutely ill foxes are loss of appetite, overexcitability, muscle cramps, fever, vomiting, and diarrhea. In recent years, CAdV-1 still has a high prevalence in different species of foxes (5-7). The virus has been identified in Italian red foxes (8), British red foxes, Korean fennec foxes (9), and Norwegian 
arctic foxes (10). Although CAdV-1 has a worldwide distribution, species susceptibility to the virus varies, with silver foxes (Vulpes vulpes) being more susceptible than arctic foxes, and arctic foxes more susceptible than dogs (11).

As post-exposure therapeutic interventions are often times not highly efficacious or economically viable options, the most effective way to prevent this virus infection in foxes is through vaccination. At present, there are many vaccine products for canine adenovirus protection, but most of them are CAdV-2 based vaccines (12). However, the latest research shows that domestic dogs vaccinated with the CAdV-2 live attenuated vaccine will shed virus, which can cause result in transmission between foxes and dogs, even in vaccinated animals (13). An early live attenuated CAdV-1 vaccine prepared by Zhou et al. was demonstrated to be safe, highly immunogenic, and provided long-lasting immunity (14). However, the attenuated CAdV-1 vaccine can cause damage to the eye membranes and kidneys of vaccinated animals. Therefore, further exploration of CAdV-1 vaccines is crucial.

Here, the use of a CAdV-1 F1301 strain isolated from a naturally infected Chinese sliver fox and passaged in MDCK cells will be characterized as a potential vaccine candidate (15). This virus was inactivated by $0.2 \%$ of formaldehyde. Aluminum hydroxide was used as an adjuvant, as it has a track record of being safe, effective $(16,17)$, and induces strong immune responses (18).

\section{MATERIALS AND METHODS}

\section{Ethics Statement}

The experimental procedures outlined below were reviewed, approved, and conducted in compliance with the guidelines of the IACUC of the hosting institution. The approved research protocol number is NO.ISAPSAEC-2021-35.

\section{Virus Preparation}

Dulbecco's modified eagle's medium (DMEM) (Gibco, USA), supplemented with $100 \mathrm{U} / \mathrm{mL}$ penicillin, and $100 \mu \mathrm{g} / \mathrm{mL}$ streptomycin (Gibco USA) was used to cultivate the Madin-darby canine kidney cell line (MDCK) (ATCC, China) at $37^{\circ} \mathrm{C}$ in a $5 \%$ $\mathrm{CO}_{2}$ incubator (Thermo scientific, USA).

A CAdV-1 F1301 strain, isolated from a naturally infected sliver fox, was cultured in MDCK cells and used as a vaccine candidate strain. Briefly, confluent MDCK monolayers were washed with PBS and inoculated with CAdV-1 F1301 suspended in DMEM (Invitrogen, Carlsbad, CA, USA). After $36 \mathrm{~h}$ at $37^{\circ} \mathrm{C}$ under $5 \% \mathrm{CO}_{2}$, the culture medium was changed to remove noninfectious viruses. The infected cells were maintained at $37^{\circ} \mathrm{C}$ and $5 \%(\mathrm{v} / \mathrm{v}) \mathrm{CO}_{2}$. Cytopathic effects (CPEs) were evaluated daily by light microscopy (Nikon, Japan). When the CPE occurred in more than $90 \%$ of cells, the flask was subjected to 3 freeze/thaw cycles between -20 to $4^{\circ} \mathrm{C}$. The cells were then collected and centrifuged at $4^{\circ} \mathrm{C}, 10,000 \times \mathrm{g}$ for $15 \mathrm{~min}$ to collect the clarified virus-containing fluid. The titers were determined using the method of Reed and Muench and expressed as TCID 50 units $/ \mathrm{mL}$.

\section{Vaccine Preparation}

The titers of CAdV-1 F1301 propagated in MDCK cells were determined to be $>10^{7} \mathrm{TCID}_{50} / \mathrm{mL}$ was inactivated with $0.2 \%(\mathrm{v} / \mathrm{v})$ formaldehyde at $37^{\circ} \mathrm{C}$ for $24 \mathrm{~h}$. The vaccine containing aluminum hydroxide gel (Changchun Huayi Biological Technology Co., Ltd.) was prepared by adding the adjuvant to a suspension of inactivated virus mixed in a volume ratio of $5: 2$. The $\mathrm{pH}$ value was adjusted to $7.2-7.4$, and stored at $4{ }^{\circ} \mathrm{C}$. The commercial $\mathrm{CAdV}-2$ live attenuated vaccine was purchased from Jilin Teyan Biotechnology Co., Ltd.

\section{Physical Properties and Sterility Testing of Inactivated CAdV-1 Vaccines}

The inactivated CAdV-1 vaccines samples were inoculated on thioglycolate medium (TG) and $0.5 \%$ broth medium, and the two media were cultured at 37 and $25^{\circ} \mathrm{C}$, respectively. Bacterial growth was determined after 3-5 days incubation (19).

\section{Verification of Virus Inactivation}

Aliquots of inactivated virus solution were batch tested to validated inactivation. Vaccine virus preparations were used to inoculate MDCK cells; the control group receives the same dose of CAdV-1 live virus. An indirect immunofluorescence test (IFA) was used to detect infectious virus.

\section{Determination of Formaldehyde Content in Inactivated Virus Solution}

A formaldehyde concentration standard of $0.1 \mathrm{mg} / \mathrm{mL}$ was used to compare experimental data. The inactivated virus solution was diluted 10 times with ultrapure water as the sample to be tested. From each standard and test sample, $0.5 \mathrm{~mL}$ was removed and added to the test buffer $(10.0 \mathrm{~mL}$ of acetic acidammonium acetate buffer, $10.0 \mathrm{~mL}$ of acetone solution), and incubated at $60^{\circ} \mathrm{C}$ for $15 \mathrm{~min}$, then rinsed with cold water for $5 \mathrm{~min}$, and let stand at room temperature for $20 \mathrm{~min}$. Absorbance at a wavelength of $410 \mathrm{~nm}$ was measured and calculated by the automated microtiter plate reader (Bio-Rad, USA). Formaldehyde solution $(40 \%)$ content $\%(\mathrm{~g} / \mathrm{mL})=0.25$ $\times$ (absorbance of the sample solution to be tested/absorbance of the standard solution) $\%$.

\section{Safety Assessment of Inactivated CAdV-1 Vaccine}

The inactivated CAdV-1 vaccine (inactivated CAdV-1 + aluminum gel saline, 5:2, $3 \mathrm{~mL}$ ) and PBS (PBS + aluminum gel saline, 5:2, $3 \mathrm{~mL}$ ) was twice injected into the hind limbs of healthy silver foxes in the immunized group and control group $(n=6)$ with a 2 -week interval. The physical condition, body temperature, and food intake of the animals were observed daily, for 10 days following immunization.

\section{Evaluation of Inactivated CAdV-1 Vaccine Immunogenicity in Fox} Animal Experiments

Healthy silver foxes aged 2-3 months, provided by the Zuojia Experimental Animal Base of the Specialty Research Institute, all animals were negative for CAdV-1, CDV, and CPV antigens 
and antibodies by PCR and serum neutralization tests, were used for vaccination and challenge studies. Healthy silver foxes $(n=$ 24, Negative to CAdV-1 and CAdV-2) at the age of 2-3 months were selected and randomly divided into 3 groups, the inactivated CAdV-1 vaccine group, the commercial CAdV-2 attenuated live vaccine group, and control group, with 8 animals in each group. The commercial CAdV-2 attenuated live vaccine (in $1 \mathrm{~mL}$ ) and inactivated CAdV-1 F1301 with aluminum hydroxide-adjuvant vaccines $(5: 2$, in $1 \mathrm{~mL})$ were intramuscularly injected into silver foxes. The foxes of the control group were injected with the same volume of PBS with aluminum gel saline. Vaccines (5:2, in $1 \mathrm{~mL}$ ) were given intramuscularly, twice, with a 2-week interval. On days $0,7,14,21$, and 30 days post vaccination, blood was collected from all foxes. From 0 to 7 days post-vaccination, rectal swabs were collected from all animals, mixed with $0.5 \mathrm{~mL}$ PBS, and stored at $-20^{\circ} \mathrm{C}$. On the 30 th day post-vaccination, all foxes were challenged with the CAdV-1 F1301 strain. An inoculum containing $100 \mathrm{LD}_{50}\left(10^{5} \mathrm{TCID}_{50}\right)$ of $\mathrm{CAdV}-1$ was injected intramuscularly into the hind limbs of all animals. Within $0-7$ days post challenge, rectal swabs were collected from all foxes daily, mixed with $0.5 \mathrm{~mL}$ PBS and stored at $-20^{\circ} \mathrm{C}$.

\section{Detection of CAdV-1 Serum Neutralizing Antibodies}

The "fixed virus-diluted serum" method was used to detect the CAdV-1 neutralizing antibody titers in silver fox serum. The original serum of the three group were added in the first row of the plate. $100 \mu \mathrm{l}$ of original serum was diluted with double dilutions starting from 1: 2 with the row. $50 \mu$ l of 100 TCID $_{50}$ was added into each well of the plate. The plate was incubated at $37^{\circ} \mathrm{C}$ for $1 \mathrm{~h}$. The solution in the plate was transferred to another cell culture plate with confluent monolayer MDCK cells. $150 \mu \mathrm{l}$ of maintenance media was added into each well of the plate. The cell plate was cultured for $96 \mathrm{~h}$. The cytopthic effect in each well was observed and the Reed-Muench method was used to calculate serum titer.

\section{Detection of Virus Shedding Post-vaccination}

The Easy pure viral DNA Kit (Beijing Quanshi Jin Company) was used to extract CAdV-1 DNA from silver fox rectal swabs, and qPCR was used to detect CAdV DNA. Primers used to detect the CAdV DNA are presented in Table 1. The thermocycling profile for the reaction consisted of an initial denaturation/enzyme activation at $95^{\circ} \mathrm{C}$ for $10 \mathrm{~min}$ was followed by 45 cycles of denaturation at $95^{\circ} \mathrm{C}$ for $15 \mathrm{~s}$, annealing at $60^{\circ} \mathrm{C}$ for $1 \mathrm{~min}$, and extension at $72^{\circ} \mathrm{C}$ for $1 \mathrm{~min}$, with a final extension at $72^{\circ} \mathrm{C}$

TABLE 1 | Primers used to detect the CAdV DNA content.

\begin{tabular}{ll}
\hline Primer (probe) & Sequence (5' to 3') \\
\hline CAdV-F & 5'-AGTAATGGAAACCTAGGGG-3' \\
CAdV-R & 5'-TCTGTGTTCTGTCTTGC-3' \\
CAdV-1 & 5'-FAM-TCAATCGTCTCAACTAAATGCCGTG-BHQ1-3' \\
CAdV-2 & 5'-TxR-TCAGTCATCTCAGCTCAATGCCGTG-BHQ1-3'
\end{tabular}

for $5 \mathrm{~min}$. Any CT value after 40 cycles was considered to be non-specific, and therefore negative.

\section{Changes in Body Temperature and Survival Rates Following Vaccination and Lethal Challenge}

After the challenge experiment, the rectal temperature of all animals was monitored at 9 am and 5 pm for 10 days. Group survival rates were calculated everyday at 10 days post challenge.

\section{Statistics}

All experimental data were analyzed using SPSS 20.0 software. The body temperature, serum neutralizing antibody titers, and qPCR data were analyzed by One-way ANOVA, and the results were expressed as Mean \pm SE. * indicates significant difference $(P$ $<0.05)$, ${ }^{* *}$ indicates extremely significant difference $(P<0.01)$.

\section{RESULTS}

\section{The Quality Test of the Fox Inactivated CAdV-1 Vaccine}

The titer of CAdV-1 for vaccine construction was $10^{7.8} \mathrm{TCID}_{50}$. The inactivated CAdV-1 vaccine with aluminum hydroxide gel adjuvant was light red suspension after being stored for a month at $4^{\circ} \mathrm{C}$, with a small amount of milky white precipitated. There was no bacterial growth in the two mediums, indicating that the inactivated $\mathrm{CAdV}-1$ vaccine with aluminum hydroxide gel adjuvant was sterilized. The positive control group showed a lot of green fluorescence; the inactivated CAdV-1 group showed no fluorescence; the negative control group did not show fluorescence (Figure 1A). The formaldehyde content in the inactivated antigen prepared meeted the standard and could be applied to animal experiments.

\section{Safety of Inactivated CAdV-1 Vaccine in Animals}

Clinical signs and food intake of silver foxes receiving either the inactivated $\mathrm{CAdV}-1$ or live attenuated $\mathrm{CAdV}-2$ vaccines appeared healthy and normal, and no apparent differences were observed between the two groups. Rectal body temperatures of animals receiving the inactivated $\mathrm{CAdV}-1$ vaccine was higher than that in the control group on the 1 st and 2 nd day after vaccination, which then stabilized and returned to normal by day 3 (Figure 1B). The results indicate that the inactivated $C A d V-1$ vaccine was safe.

\section{Immunogenicity of Inactivated CAdV-1 Vaccine in Animals}

At all time-points tested, the virus neutralizing antibody (VNA) titers of both groups of vaccinated animals were significantly higher than control group $(P<0.01)$, although no significant difference was observed between the two groups (Figure 1C).

\section{Virus Shedding Following Vaccination and Lethal CAdV-1 Challenge}

From 1 to $4 \mathrm{dpv}$, the viral DNA loads of CAdV-2 in rectal swabs of animals in the live vaccine group were higher than those of 


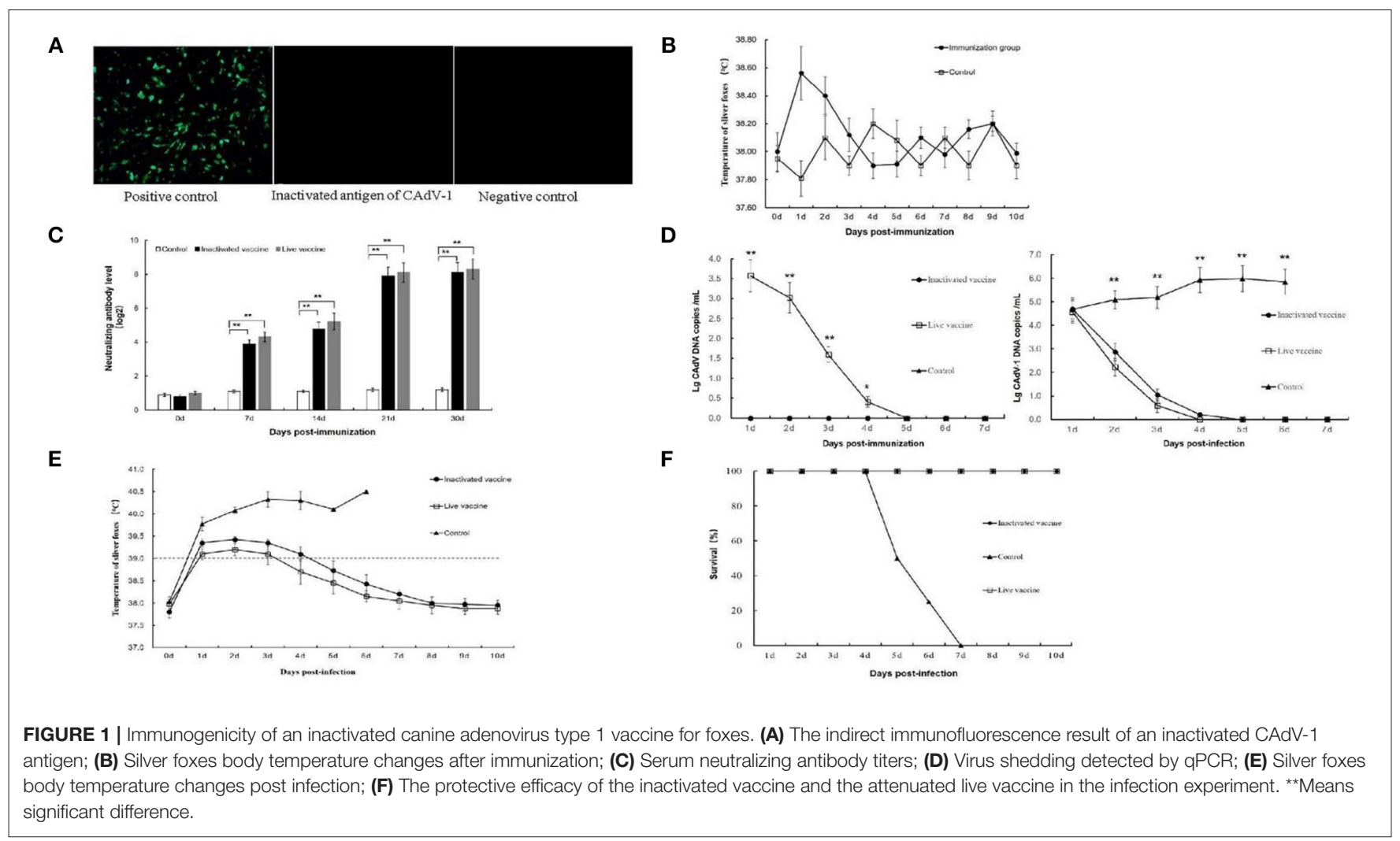

the inactivated CAdV-1 vaccine group $(P<0.05)$. No viral DNA of CAdV-1 was detected in animals that received the inactivated $\mathrm{CAdV}-1$ vaccine, indicating that the inactivated $\mathrm{CAdV}-1$ vaccine virus was not shed (Figure 1D). From 1-3 days post infection (dpi), the virus shedding ocured and decreased with the time in both live CAdV-2 vaccine group and inactivated CAdV-1 vaccine group. Through $5 \mathrm{dpi}$, rectal swabs in the both groups were negative for CAdV-1 DNA.

\section{Changes in Body Temperature and Survival Rate Following Lethal CAdV-1 F1301 Challenge}

On the first day, the body temperature of the foxes in the control group increased to above $39.5{ }^{\circ} \mathrm{C}$ and continued to rise until all animals succumbed to infection. The body temperature of the foxes in both vaccinated groups increased above $39^{\circ} \mathrm{C}$ and dropped below $39^{\circ} \mathrm{C}$ on the 4 and $5 \mathrm{dpi}$, respectively. On $6 \mathrm{dpi}$, the body temperature of both the live and inactivated vaccine groups returned to normal (Figure 1E).

In control group, all foxes succumbed by $7 \mathrm{dpi}$, and the mortality rate was $100 \%$. In inactivated and commercial CAdV-1 vaccine groups, all animals survived until the end of the study. Both vaccines were successful at mitigating the effects of lethal virus challenge (Figure 1F).

\section{DISCUSSION}

In contrast to many viruses that are tightly host restricted, CAdV1 infects a wide range of animals, include dogs and foxes. In recent years, CAdV-1 has been highly prevalent in different species of foxes worldwide (5-7), including Italian red foxes (8), British red foxes, Korean fennec foxes (9), and Norwegian arctic foxes (10). Unfortunately, CAdV-1 is not sensitive to most disinfectants, so prevention of transmission via fomites is exceptionally challenging. Therefore, the most effective way to prevent infection of foxes is vaccination. At present, there are many vaccine products for canine adenovirus protection, but most of them protect against CAdV-2. However, the latest research shows that domestic dogs vaccinated with the CAdV2 attenuated live vaccine will shed virus. This may lead to cross species infection (which may be sub-clinical) from dogs to foxes that come into direct contact with each other. Furthermore, only CAdV-1, but not CAdV-2, was detected in dead animals (13). Therefore, the CAdV-2 attenuated live vaccine still has risks, and seems to lack cross-protective efficacy against CAdV-1 infection. The fox CAdV-1 F1301 strain isolated from a naturally infected Chinese silver fox is a highly stable strain and can be cultured to very high titers $\left(10^{8} \mathrm{TCID}_{50} / \mathrm{ml}\right)$ in MDCK cells. CAdV-1 F1301 strain meets the standards of "Chinese Veterinary Medicine," which makes it a desirable platform from which to construct inactivated $\mathrm{CAdV}-1$ vaccines. According to the physical characteristics of the CAdV-1 virus, and referring to the state of "Chinese veterinary pharmacopeia," a final concentration of $0.2 \%$ formaldehyde was used to completely inactivate the virus.

The virus strain of the inactivated vaccine must be completely inactivated and its immunogenicity must be preserved as much as possible. The selection of an optimal adjuvant is essential when seeking to enhance and direct the immune response to vaccination. The aluminum hydroxide gel is stable, inexpensive, 
and is already in wide use worldwide. In addition, aluminum hydroxide gel can adsorb antigens on its surface to enhance immunity. Therefore, aluminum hydroxide as the adjuvant is well suited to compensate for the typical poorly immunogenic nature of inactivated vaccines (20).

The inactivated CAdV-1 vaccine developed here was safe and efficacious in animals, complied with the sterile standard, and the residual formaldehyde meets the standards of "Chinese Veterinary Medicine." The physical condition and appetite of the vaccinated animals were normal, and a mild transient spike in body temperature, which lasted 2 days, was observed. In addition, detection of CAdV DNA in rectal swabs of vaccinated animals revealed shedding of the commercial live-attenuated vaccine (1$4 \mathrm{dpv})$. No virus DNA was detected in the inactivated CAdV-1 vaccine group $(P<0.01)$.

The neutralizing antibody titers of the inactivated CAdV1 vaccinated animals reached a peak on the $30 \mathrm{dpv}$, with no statistical difference being observed between the live-attenuated and inactivated vaccine groups $(P>0.05)$. After inoculation with a lethal CAdV-1 challenge, all silver foxes were shedding virus within 1-3 days and did not until the fifth day. The body temperature of silver foxes initially increased, decreased to normal by $6 \mathrm{dpv}$. The above experimental results demonstrate the safety and efficacy of the inactivated CAdV- 1 vaccine, and it was similar to the commercial vaccine, both of which exhibited protective efficacy in silver foxes.

In summary, we created a new inactivated CAdV- 1 vaccine based on the F1301 strain that was isolated from a naturally infected Chinese sliver fox. The inactivated CAdV-1 vaccine with the aluminum hydroxide adjuvant was safer in silver foxes

\section{REFERENCES}

1. Wang SJ, Wang CS, Ren X, Xue WJ, He HJ, Zhu YZ, et al. Identification of two novel linear neutralizing epitopes within the hexon protein of canine adenovirus using monoclonal antibodies. Vaccines. (2021) 9:135. doi: $10.3390 /$ vaccines 9020135

2. Jonge BD, Brantegem LV, Chiers K. Infectious canine hepatitis, not only in the textbooks: a brief review and three case reports. Vlaams Diergeneeskd Tijdschr. (2020) 89:284-91. doi: 10.21825/vdt.v89i5.16956

3. Hu RL, Huang G, Qiu W, Zhong ZH, Xia XZ, Yin Z. Detection and differentiation of CAV-1 and CAV-2 by polymerase chain reaction. Vet Res Commun. (2001) 25:77-84. doi: 10.1023/A:1006417203856

4. Oleaga A, Ana Balseiro A, Alberto Espí A, Royo LJ. Wolf (canis lupus) as canine adenovirus type $1(\mathrm{CAdV}-1)$ sentinel for the endangered cantabrian brown bear (ursus arctos arctos). Transbound Emerg Dis. (2021). doi: 10.1111/tbed.14010. [Epub ahead of print].

5. Hechinger S, Scheffold S, Hamann HP, Zschöck M. Detection of canine adenovirus 1 in red foxes (Vulpes vulpes) and raccoons (Procyon lotor) in Germany with a TaqMan real-time PCR assay. J Vet Diagn Invest. (2017) 29: 741-6. doi: 10.1177/1040638717712331

6. Thompson H, O'keeffe AM, Lewis JCM, Stocker LR, Laurenson MK, Philbey AW. Infectious canine hepatitis in red foxes (vulpes vulpes) in the United Kingdom. Vet Rec. (2014) 166:111-4. doi: 10.1136/vr.b4763

7. Walker D, Abbondati E, Cox AL, Mitchell GBB, Pizzi R, Sharp CP, et al. Infectious canine hepatitis in red foxes (Vulpes vulpes) in wildlife rescue centres in the UK. Vet Rec. (2016) 178:421. doi: 10.1136/vr.103559

8. Balboni A, Verin R, Morandi F, Poli A, Prosperi S, Battilani M. Molecular epidemiology of canine adenovirus type 1 and type 2 in free-ranging than the commercially available live vaccines. Therefore, the foxderived CAdV-1 F1301 strain appears to be a promising vaccine platform candidate to protect against natural infection in both farms and the wild.

\section{DATA AVAILABILITY STATEMENT}

The raw data supporting the conclusions of this article will be made available by the authors, without undue reservation.

\section{ETHICS STATEMENT}

The animal study was reviewed and approved by IACUC of the hosting institution.

\section{AUTHOR CONTRIBUTIONS}

YF and JSu contributed equally to conduct and write this manuscript. YF, JSu, and SL conduct the construction of CAdV-1 inactivated vaccine. YF, JSu, XD, and LZ conducted the evaluation of safety and immunogenicity of CAdV-1 inactivated vaccine in silver fox. JSh, $\mathrm{XD}$, and LZ help to assist the experiment and correct the manuscript. XY and YZ provide grant for this experiment and review this manuscript. All authors contributed to the article and approved the submitted version.

\section{FUNDING}

This work was supported by grants from the Science and Technology Department of Jilin province (20200402045NC). red foxes (Vulpes vulpes) in Italy. Vet Microbiol. (2013) 162:551-7. doi: 10.1016/j.vetmic.2012.11.015

9. Choi JW, Lee HK, Kim SH, Kim YH, Lee KK, Lee MH, et al. Canine adenovirus type 1 in a fennec fox (Vulpes zerda). J Zoo Wildl Med. (2014) 45:947-50. doi: 10.1638/2013-0286.1

10. Balboni A, Tryland M, Mørk T, Killengreen ST, Fuglei E, Battilani M. Unique genetic features of canine adenovirus type 1 (CAdV-1) infecting red foxes (Vulpes vulpes) in northern Norway and arctic foxes (vulpes lagopus) in Svalbard. Vet Res Commun. (2019) 43:67-76. doi: 10.1007/s11259-019-09746-y

11. Sun J, Qin FY, Zhu YZ, Xue XH, Wang Y, Lian SZ, et al. Comparison of the pathogenicity of canine adenovirus type 1 to dogs, Arctic fox and silver fox. China Veterinary Science. (2019) 49:625-31. doi: 10.16656/j.issn.1673-4696.2019

12. Tamukai K, Shohei M, Kurihara R, Shimoda H, Mitsui I, Maeda K, et al. Molecular evidence for vaccine-induced canine distemper virus and canine adenovirus 2 coinfection in a fennec fox. J Vet Diagn Invest. (2020) 32:598603. doi: $10.1177 / 1040638720934809$

13. Dowgier G, Lahoreau J, Lanave G, Losurdo M, Varello K, Lucente MS, et al. Sequential circulation of canine adenoviruses 1 and 2 in captive wild carnivores, France. Vet Microbiol. (2018) 221:67-73. doi: 10.1016/j.vetmic.2018.05.025

14. Zhou XY, Yang DJ. Clinical application effect of ICH attenuated cell vaccine for canine infectious hepatitis. Raising A Dog. (1992) 8:22-3.

15. Zhu YZ, Sun J, Yan MH, Lian SZ, Hu B, Lv S, et al. The biological characteristics of the canine adenovirus type 1 from fox and the transcriptome analysis of the infected MDCK cell. Cell Biol Int. (2021) 45:936-47. doi: $10.1002 /$ cbin. 11537 
16. Mair KH, Koinig H, Gerner W, Höhne A, Bretthauer J, Kroll JJ, et al. Carbopol improves the early cellular immune responses induced by the modified-life vaccine Ingelvac PRRS MLV. Vet Microbiol. (2015) 176:352-7. doi: 10.1016/j.vetmic.2015.02.001

17. Xu YX, Wang Q, Wei BW, Huang XB. Enhanced immune responses against japanese encephalitis virus infection using Japanese encephalitis liveattenuated virus adjuvanted with montanide GEL 01 ST in mice. Vector Borne Zoonotic Dis. (2019) 19:835-43. doi: 10.1089/vbz.2018.2419

18. Coffman RL, Sher A, Seder RA. Vaccine adjuvants: putting innate immunity to work. Immunity. (2010) 33:492-503. doi: 10.1016/j.immuni.2010. 10.002

19. Li XT, Liu H, Liu S, Hu B, Wang Y, Lu RG, et al. Study on the immunogenicity and protective effect of mink pseudorabies virus DL14/08 strain. Chin J Prev Vet Med. (2017) 39:583-8. doi: 10.3969/j.issn.1008-0425.2017.07.15

20. Little SF, Ivins BE, Webster WM, Norris SL, Andrews GP. Effect of aluminum hydroxide adjuvant and formaldehyde in the formulation of rPA anthrax vaccine. Vaccine. (2007) 25:2771-7. doi: 10.1016/j.vaccine.2006.12.043
Conflict of Interest: The authors declare that the research was conducted in the absence of any commercial or financial relationships that could be construed as a potential conflict of interest.

Publisher's Note: All claims expressed in this article are solely those of the authors and do not necessarily represent those of their affiliated organizations, or those of the publisher, the editors and the reviewers. Any product that may be evaluated in this article, or claim that may be made by its manufacturer, is not guaranteed or endorsed by the publisher.

Copyright (C) 2022 Fu, Sun, Lian, Deng, Zhang, Shao, Yu, Yan and Zhu. This is an open-access article distributed under the terms of the Creative Commons Attribution License (CC BY). The use, distribution or reproduction in other forums is permitted, provided the original author(s) and the copyright owner(s) are credited and that the original publication in this journal is cited, in accordance with accepted academic practice. No use, distribution or reproduction is permitted which does not comply with these terms. 